\title{
La grande vitesse en Limousin. Une histoire de rendez-vous manqués
}

High Speed in the Limousin region. A story of missed connections

\section{Pascal Desmichel}

\section{OpenEdition}

\section{Journals}

Édition électronique

URL : https://journals.openedition.org/rhcf/1550

DOI : 10.4000/rhcf.1550

\section{Éditeur}

Rails \& histoire

\section{Édition imprimée}

Date de publication : 1 septembre 2012

Pagination : 175-190

ISSN : 0996-9403

\section{Référence électronique}

Pascal Desmichel, «La grande vitesse en Limousin. Une histoire de rendez-vous manqués », Revue d'histoire des chemins de fer [En ligne], 42-43 | 2012, mis en ligne le 14 novembre 2014, consulté le 22 avril 2022. URL : http://journals.openedition.org/rhcf/1550; DOI : https://doi.org/10.4000/rhcf.1550 
Pascal DESMICHEL

\section{La grande vitesse en Limousin Une histoire de rendez-vous manqués}

\section{Le Limousin, une « île continentale » et pourtant centrale}

Le Limousin est un territoire paradoxal. La région est souvent volontiers considérée par les géographes comme " périphérique " eu égard à son faible poids démographique, à la valeur de son produit intérieur brut, au rôle mineur de son industrie, à son enclavement. Nous sommes ici en présence du stéréotype de l'espace "profond ", du moins dans ses représentations (la réalité serait à nuancer mille fois). Cet isolement a fait dire aux géographes de l'université de Limoges que le Limousin était en quelque sorte une "île continentale ". Pourtant la région dispose paradoxalement d'une situation quasi centrale au sein de l'hexagone qui a constitué, longtemps (avant l'Europe), l'échelle de référence de l'aménagement du territoire. La situation limousine au sein du "Centre-Ouest ", entre océan et Massif central, aux portes du Sud-Ouest, à mi-chemin entre la capitale parisienne et la frontière espagnole, n'était-elle pas finalement comparable à l'espace carrefour qu'est devenue la Bourgogne? De la même manière que Dijon est devenue le point de passage obligé vers le grand Sud-Est (les voies alpines et la Suisse...), Limoges n'était-elle pas potentiellement promise à devenir le passage obligé vers le grand Sud-Ouest, qu'il s'agisse de la grande Aquitaine, des Pyrénées ou de l'Espagne ? Que s'est-il 
passé pour que le Limousin rate une telle opportunité ? Pourquoi aujourd'hui encore le TGV est-il presque absent ${ }^{1}$ ? La grande vitesse est un sujet particulièrement sensible en Limousin, et ce d'autant plus que le projet de ligne Poitiers - Limoges est venu le ranimer, sur fond de discordes. À l'heure d'une possible intégration de l'espace régional au réseau européen à grande vitesse, que se passe-t-il donc pour que le projet soulève une telle opposition, ou du moins fasse couler autant d'encre ? Quels sont les arguments développés par les pro- et anti-TGV ? Quels sont les risques encourus ?

Pour répondre à cet ensemble de questions, pour comprendre cette série de rendez-vous manqués avec la grande vitesse, nous nous sommes appuyés sur plusieurs types de sources. Les monographies historiques et les études de géographie régionale ont d'abord permis de mettre dans son contexte le chemin de fer limousin. La presse ferroviaire et ferroviphile, à travers ses éditions emblématiques (La Vie du rail, Le Cabri...), a également contribué à situer avec précision des événements économiques et politiques. Pour saisir au mieux les manifestations d'approbation ou de désaccord avec le projet de TGV Poitiers - Limoges, la presse locale ainsi que les sites et forums internet (lieux d'expression majeure de l'opinion publique) ont été largement consultés. Des visites de terrain sur le tracé annoncé de la ligne à grande vitesse (LGV) ont permis de compléter et illustrer les formes d'expression des habitants. Le tout s'est nourri d'un va et vient constant avec un corpus théorique relevant pour une large part des travaux de l'AHICF.

Nous adopterons une progression historique qui s'avère - comme très souvent - une manière efficace d'éclairer les jeux et enjeux d'aujourd'hui.

\section{Premier rendez-vous manqué : Limoges au cœur des grands itinéraires (xIX ${ }^{\mathrm{e}}$ siècle - entre-deux-guerres)}

Il est d'abord important et intéressant de rappeler que, pour qu'il y ait grande vitesse, il faut d'abord de grands itinéraires qui s'avèrent tous, à quelques exceptions près, hérités du XIX ${ }^{\mathrm{e}}$ siècle. Autrement dit, la géographie de la grande vitesse existe déjà à cette époque. Le réseau TGV du Xxi ${ }^{\mathrm{e}}$ siècle ne s'appuie-t-il pas en majorité sur le réseau classique hérité, bien davantage en dernière analyse que sur les tronçons récemment construits, les véritables " lignes TGV » ? Cela veut donc dire que les itinéraires construits au XIX ${ }^{e}$ siècle ont conditionné, façonné, dessiné, la géographie ferroviaire contemporaine et,

1- Une rotation quotidienne par TGV entre Brive et Lille existe depuis $2007 \ldots$ financée en partie par la collectivité. 
peut-être, la géographie tout court (que serait, là encore, devenue Dijon sans fonction de carrefour ? En Limousin, à une échelle plus modeste, Brive auraitelle aussi vite supplanté Tulle sans le développement de son étoile ferroviaire ?, etc.). Certes, nous savons à quel point les contraintes topographiques ont pu jouer un rôle, qui est incontestable, dans les choix et les qualités des dessertes. Mais cela ne suffit pas à tout expliquer ; il existe des passages "naturels " qui semblent avoir - provisoirement - été oubliés par l'histoire politique avant de revenir dans l'actualité des décennies plus tard. C'est en ce sens que l'histoire nous apporte des éclairages passionnants.

Une lecture d'ouvrages de référence révèle que le sort attribué à Limoges par les grandes compagnies ferroviaires a été flatteur, mais sur le papier. Au début des années 1850, la célèbre et éphémère compagnie du " grand central » a des ambitions pour Limoges dont elle fait son point de passage obligé entre Paris et Madrid via Agen, Tarbes, Saragosse, avec le franchissement des Pyrénées par - déjà - un tunnel sous le Somport et Canfranc ${ }^{2}$. Plus tard, alors que le Limousin est devenu le domaine quasi exclusif de la Compagnie du Paris-Orléans, l'ère des grands projets transcontinentaux de l'entre-deuxguerres se montre une nouvelle fois favorable au développement du territoire limousin et de sa capitale. Un axe "Suisse - Océan " est évoqué pour relier Bordeaux - Limoges - Montluçon - Lyon - Genève, avec notamment la création d'un nouveau tronçon Limoges - Saint-Germain-des-Fossés venant supplanter l'existant, dont les caractéristiques sont jugées trop médiocres. Le "Bordeaux - Odessa " (ou "Atlantique - mer Noire ", " ligne du 45 parallèle », ou encore « barrière antigermanique "...) est basé sur ce même principe de liaison transversale d'envergure internationale avec la promotion d'une voie Bordeaux - Limoges - Lyon - Turin - Milan - Venise - Trieste - Belgrade - Bucarest. La dégradation du contexte politique international, la crise des grandes compagnies mettent un terme à ces ambitions déjà européennes. Nouveaux tronçons, électrification, suppressions de rebroussements ne sont pas réalisés.

C'est finalement - et l'histoire l'oublie - la création d'une ligne entre Brive et Montauban qui va, tardivement et presque par hasard, faire émerger en 1891 un itinéraire direct entre Paris et ce qui est alors somme toute une très modeste et peu dynamique ville de Province, Toulouse (149 791 habitants en 1891, 149576 en 1911)3. La radiale Paris - Limoges - Brive - Toulouse

2- Réalisé près de 75 ans plus tard, l'itinéraire n’aura plus la même légitimité. Voir DESMICHEL, 2008.

3- Dans le même laps de temps, Limoges gagne 20000 habitants, passant de 72000 à 92000 habitants. 
constitue avec sa voie double (très tôt électrifiée), une des artères majeures du réseau ferré national.

Finalement, donc, la géographie du chemin de fer limousin n’est pas celle qui avait été pensée à l'origine ; elle ne correspond pas en effet aux ambitions des compagnies du grand central et du PO, ou même du ClermontTulle (fig. 1).

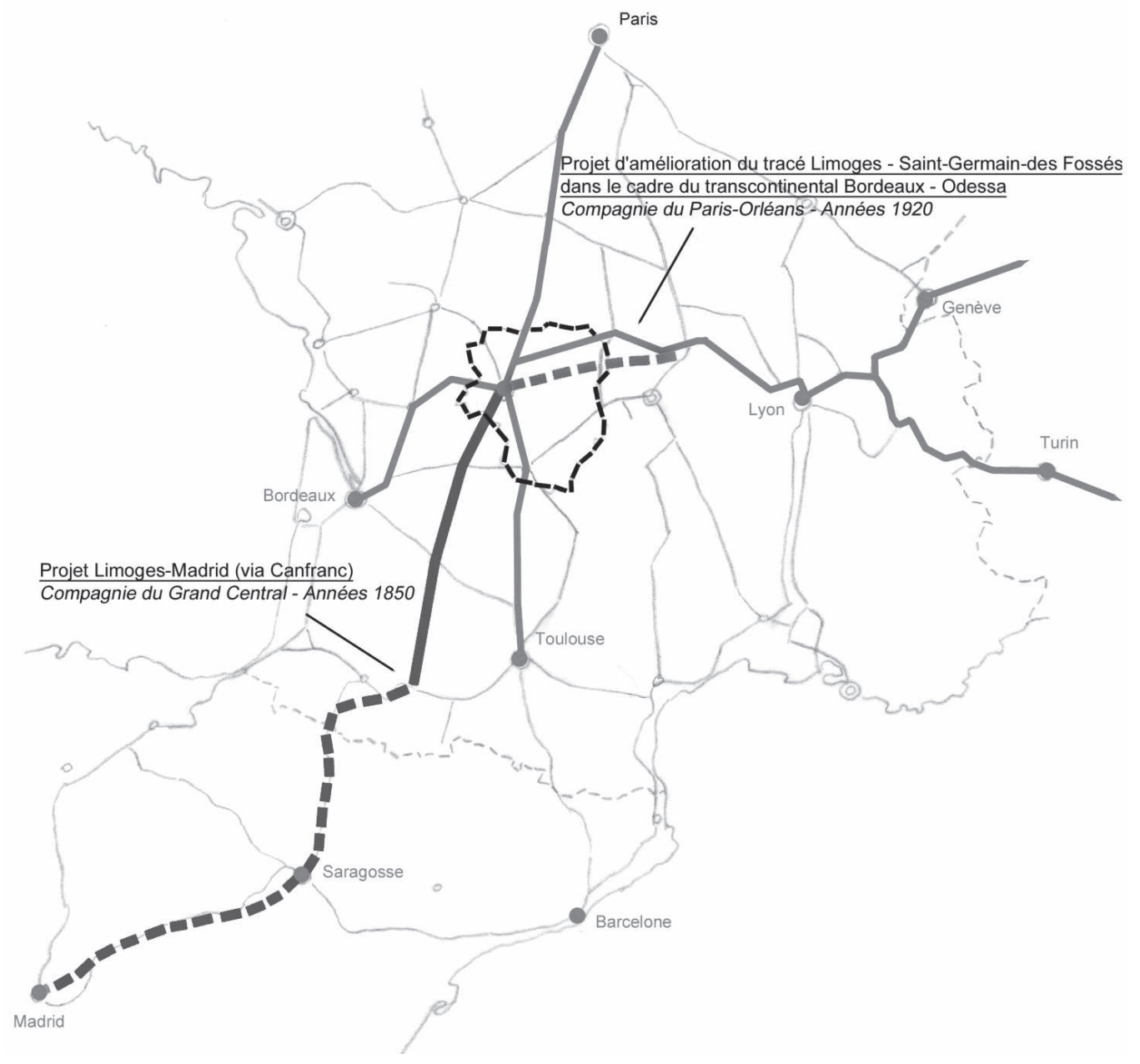

Figure 1. Les grands projets du Limousin : des rêves à la réalité. 


\section{Des années 1960 à la fin du Capitole}

En dépit d'un premier rendez-vous manqué, qui aurait dû permettre la naissance d'un grand carrefour ferroviaire, le Limousin n'est pas encore oublié par le progrès dans ce domaine et devient même dans les années 1960 l'une des premières régions françaises à bénéficier des débuts de la grande vitesse. L'emblématique Capitole et, dans une moindre mesure, les ETG / RTG qui circulent sur les liaisons transversales s'inscrivent dans l'épopée du chemin de fer moderne ${ }^{4}$. Mais que s'est-il donc passé pour que tout bascule à peine deux décennies plus tard?

\section{« L'âge d'or » des années 1960 à 1980}

1967 marque la naissance du premier train européen ${ }^{5}$ " à grande vitesse » circulant à $200 \mathrm{~km} / \mathrm{h}$ en vitesse commerciale ; le Capitole (du nom de la célèbre place toulousaine) annonce le désir de modernité de la SNCF avec un matériel roulant très confortable et l'affichage de ses ambitions ; le " design " et la nouvelle livrée rouge s'affirment dans le paysage. Le train conquérant entend montrer ses atouts face à un secteur aérien qui gagne des parts de marché. Limoges et le Limousin s’avèrent les premiers bénéficiaires de ces débuts de la course à la grande vitesse. C'est en effet à travers les plaines de Sologne et du Berry que les temps de parcours sont considérablement diminués grâce à l'aménagement de sections franchies à $200 \mathrm{~km} / \mathrm{h}$ (entre Orléans et Vierzon en particulier). La capitale limousine peut alors se targuer, bien plus que Toulouse, de figurer parmi les cinq villes les mieux desservies de France depuis Paris, avec une vitesse commerciale des liaisons supérieure à celle qui concerne des métropoles telles que Lille, Strasbourg, Marseille, Grenoble et bien d'autres.

Le Capitole obtient le label Trans Europ Express (TEE) en 1970 et entre alors dans le cercle fermé et prestigieux des grands trains européens. Outre cette grande liaison radiale parcourue deux fois par jour par les rames rouges,

4- ETG : élément à turbine à gaz ; RTG : rame à turbine à gaz. Les ETG ont été mis en service en 1971 sur la ligne Paris-Caen-Cherbourg, puis envoyés sur les lignes Lyon-Grenoble, Valence - Grenoble - Chambéry - Genève et Lyon - Chambéry - Annecy. Les RTG, construits à partir de 1972, sont aptes à rouler à $160 \mathrm{~km} / \mathrm{h}$; ils ont été employés sur les transversales LyonStrasbourg et Lyon-Nantes, puis sur Lyon-Bordeaux et Bordeaux-Toulouse. Ils ont remplacé les ETG sur les lignes Paris-Cherbourg et Paris-Deauville-Cabourg et ont roulé sur les lignes Lyon-Grenoble, Bordeaux-La Rochelle, Paris-Calais, Caen-Tours, Tours-Nantes, Nantes-Le Mans, Paris-Boulogne, Paris-Laon, Besançon-Clermont et Lyon-Clermont. Les dernières rames ont été retirées du service en 2004 [N.d.l.R.].

5 - Le premier train à grande vitesse au monde est japonais... 
le Limousin bénéficie sur ses lignes transversales d'un matériel à la pointe du progrès, avec les ETG puis RTG considérés comme les ancêtres du TGV. Ces rames circulent en assurant des relations d'envergure comme Genève (ou Turin) - Lyon - Bordeaux, ou Vichy - Nantes. S'il n'est pas possible de parler ici de grande vitesse au sens contemporain du terme, le niveau du confort et des prestations offertes en font un service ferroviaire de grande qualité pour l'époque. Le Limousin s'inscrit dans un réseau encore homogène en matière de desserte nationale, voire européenne. Évoquer un quelconque enclavement des villes limousines est objectivement difficile au regard de la situation générale. Avec un réseau encore bien maillé ${ }^{6}$ une radiale particulièrement compétitive, des transversales parcourues par des trains confortables conduisant à l'océan ou aux Alpes, le Limousin n'a finalement pas le sort peu enviable qu'on lui attribue généralement.

\section{Le tournant raté des années 1990}

De la fin des années 1960 aux premiers signes avant-coureurs du déclin (le Capitole perd son label TEE dès 1984), cette sorte "d'âge d'or " du chemin de fer limousin ne dure qu'une petite vingtaine d'années. Que s'est-t-il donc passé ? Plusieurs hypothèses peuvent être avancées par le géographe qui s'attache à placer le contexte régional dans la perspective plus globale de la mutation des sociétés et des territoires.

\section{Autopsie d'un échec programmé (?)}

Évoquons d'abord le phénomène croissant de la métropolisation qui, comme le soulignent Jacques Lévy et Michel Lussault, est une «notion-clé pour comprendre l'évolution des territoires des pays économiquement avancés "; le processus d'urbanisation réduit depuis plusieurs décennies le poids relatif des villes petites et moyennes au profit des très grandes aires urbaines qui concentrent de plus en plus les réponses aux besoins des entreprises et des couches sociales les plus qualifiées. Les villes sont entrées dans une ère de

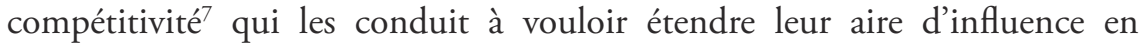
investissant dans des équipements susceptibles d'attirer ces nouvelles entreprises et de nouveaux habitants. À partir des années 1970-1980, une France post-industrielle se dessine ; le Sud-Ouest longtemps considéré comme " pauvre " (sous-industrialisé, peu urbanisé, avec une empreinte rurale très

6- Les campagnes du Massif central restent proportionnellement plus épargnées que les autres territoires français.

7- Le terme sera de plus en plus usité ; il sera plus tard question de DIACT (Délégation interministérielle à l'aménagement et à la compétitivité des territoires), de pôles de compétitivité. 
marquée) fait désormais partie des régions gagnantes. Le boom de l'activité aéronautique transforme tout particulièrement Toulouse qui, de modeste ville de province, devient une véritable métropole européenne attirant chaque année plus de 10000 nouveaux habitants. C'est toute la géographie française qui se recompose dans ce dernier quart de $\mathrm{xx}^{\mathrm{e}}$ siècle. C'est désormais à l'ouest du célèbre axe virtuel Le Havre - Marseille que la dynamique démographique et économique s'emballe. C'est dans ce laps de temps que le fossé entre "grandes villes » et " métropoles européennes en devenir » se creuse ; Limoges, comme Clermont-Ferrand ou Dijon, ne pèsent plus face à la montée en puissance des villes de l'Ouest (Rennes, Nantes) et du Sud (Bordeaux, Toulouse mais aussi Montpellier et toute sa région). Cette métropolisation est aussi bien sûr indissociable du processus de mondialisation et de la montée en puissance du transport aérien qui, en se démocratisant, bouleverse nos manières de nous déplacer. La SNCF a très tôt intégré cette nouvelle donne dans son approche stratégique au point d'en faire son leitmotiv; désormais, l'enjeu commercial de l'entreprise est d'être capable de concurrencer l'avion sur les relations de ville à ville sur des parcours de moins de trois heures.

Dans ce double contexte (dynamique de métropolisation à l'Ouest et au Sud, montée en puissance du transport aérien), le choix de Toulouse de contourner Limoges n'a rien d'imprévisible. L'ère de « l'aménagement du territoire " a cédé la place à celle de la compétitivité des territoires, et les 710000 habitants du Limousin ne pèsent politiquement comme économiquement pas lourd face aux intérêts immédiats de deux régions en pleine croissance démographique, avec à leur tête deux métropoles soucieuses de rattraper leur retard et de trouver leur place dans une Europe qui s'élargit jusquau monde ibérique $^{8}$. Toulouse veut profiter immédiatement de l'arrivée du TGV Aquitaine pour pallier - dans la mesure du possible - son enclavement. Le «schéma directeur de la grande vitesse ferroviaire $"{ }^{9}$ adopté par le gouvernement français en 1991 promet déjà aux acteurs toulousains une LGV autorisant des temps de parcours inférieurs à trois heures. Le Limousin, mais aussi tout le nord de la région Midi-Pyrénées, sont mis à l'écart ; la faiblesse démographique des départements (et de leurs villes ${ }^{10}$ ), de même que les difficultés que présente le relief semblent définitivement rédhibitoires pour un système TGV promis à un vif succès, notamment sur le marché international. Le Limousin et l'Auvergne ne

8- 1986 : entrée de l'Espagne et du Portugal dans l'Union Européenne.

9- Reprenant la plupart des projets locaux, le schéma directeur compte 16 lignes, totalisant $3172 \mathrm{~km}$ de voies.

10- La ligne ne dessert que de modestes préfecture et sous-préfectures sans importance économique notable. 
sont certes pas oubliés sur la carte du schéma directeur. Limoges et ClermontFerrand se trouvent associées autour de l'idée d'un "Y renversé " dont le principe est de créer un tronçon de LGV commun au sud de Paris avant une séparation en deux branches près de Vierzon ou Bourges ${ }^{11}$. Force est de constater que jamais les deux régions du Massif central n'ont uni leurs forces pour développer le lobby qui aurait appuyé cette solution.

"L'affaire" du POLT : la fin d'un rêve difficile à abandonner... ?

En Limousin, ce TGV « Centre » emporte difficilement l'adhésion parce que l'opinion publique et ses représentants politiques ne parviennent pas à changer de schéma (dans tous les sens du terme). Pour que s'impose un nouveau schéma géographique, il faudrait en effet adopter un nouveau schéma de pensée qui oublierait l'ère glorieuse et à peine achevée du Capitole. Or les esprits ne sont pas disposés à " faire le deuil " de cette desserte prestigieuse. Par conséquent, la stratégie de l'exécutif régional consiste à défendre et à promouvoir le principe d'un TGV Paris - Limoges - Toulouse (le projet « Palito " qui deviendra «POLT » avec l'engagement de la région centre et de sa capitale Orléans). Du matériel pendulaire d'origine italienne est testé dès 1997 sur la radiale qui souffre au sud de Limoges d'un profil plutôt médiocre dû notamment à des courbes resserrées. Les résultats s'avèrent peu concluants, la SNCF et le constructeur Alsthom se montrent de toute façon - et dès le début de l'expérience - sceptiques pour ne pas dire hostiles à la technique pendulaire. Les régions s'engagent alors dans des études d'amélioration de la ligne avec le projet de créer ponctuellement de nouveaux tronçons autorisant des accroissements significatifs de vitesse. Ce principe d'aménagement du parcours classique emporte l'adhésion; il permet des gains de temps non négligeables sans pour autant laisser à l'écart les territoires fragiles traversés (Berry, Marche...). L'État, qui s'est engagé à réaliser le TGV POLT et à le mettre en service en 2006, fait pourtant volte-face en 2003. Le gouvernement Raffarin juge le projet irréalisable ${ }^{12}$ et ne le retient pas dans le cadre du Comité interministériel d'aménagement et de développement du territoire (CIADT) du 18 décembre. Pour la première fois, des régions déposent un recours devant le Conseil d'État

11- Ce principe sera adopté et concrétisé quelques années plus tard pour les infrastructures autoroutières.

12- «La solution de TGV pendulaire, en termes de gain de temps, d'échéance de la réalisation et de la rentabilité du projet, n'était pas pertinent en termes d'investissement comme d'exploitation », explique Gilles de Robien aux présidents des régions concernées lors d'une réunion à Paris le 9 novembre 2004 (Le Monde, 13 novembre 2004). 
pour obtenir le remboursement des études menées ${ }^{13}$. Cette affaire du POLT fait grand bruit et suscite une vive émotion en Limousin où élus comme habitants se sentent abandonnés et trahis.

C'est bien une logique métropolitaine, celle qui accorde la primauté aux grands centres urbains que sont Paris et Toulouse, qui prévaut sur la desserte de départements à caractère rural tels que l'Indre, la Creuse, la Corrèze, Le Lot, l'Aveyron ou le Tarn. Le vieux rêve n'est cependant pas tout à fait abandonné ; un TGV Brive - Lille est inauguré en décembre 2007. Cette rotation quotidienne, co-financée par les régions Centre et Limousin (1 million d'euros par an pour chaque collectivité et 3 millions pour la SNCF) emprunte la ligne historique et offre pour la première fois une connexion directe avec le réseau européen à grande vitesse via Juvisy, Marne-la-Vallée et Roissy-Charles de Gaulle. La radiale Paris - Limoges - Toulouse semble quoi qu'il en soit relever du passé. La section Brive - Montauban est la plus touchée par ce remaniement progressif des dessertes ${ }^{14}$; le département du Lot souffre de ce " dépeçage » qui a tendance à faire de Brive un terminus (fig. 2). À l'opposé de la grande vitesse, la ligne sert même de cadre d'expérience pour développer un produit " petite vitesse » que la SNCF inaugure en 2010 ; il s'agit en effet de faire circuler des rames Lunéa en plein jour pour un public familial acceptant de parcourir $700 \mathrm{~km}$ en sept heures pour une somme modique de 15 euros par personne. Prendre son temps pour voyager, rechercher le confort du parcours plutôt que la vitesse coûte que coûte ; c'est aussi une tendance nouvelle qu’observe le sociologue Vincent Kaufmann ${ }^{15}$.

13- L'État a été condamné en 2008 par le Conseil d’État à rembourser 340000 euros à la région Midi-Pyrénées après l'abandon du projet. Le Conseil d'État a également rendu un arrêt favorable aux régions Centre et Limousin mais le préjudice pour manque à gagner les concernant n'a pas été retenu.

14- Une comparaison entre 1988 et 2010 des rotations quotidiennes directes entre Paris et Toulouse (desservant Cahors) fait état d'une baisse sensible des circulations directes : 4 Teoz en journée contre 6 Corail avant l'arrivée du TGV via Bordeaux, auxquelles s'ajoutent les circulations nocturnes qui permettaient aux habitants de Limoges et Brive d'accéder dans d'excellentes conditions à la capitale de Midi-Pyrénées en début de matinée.

15- KAUFMANN, 2008. 


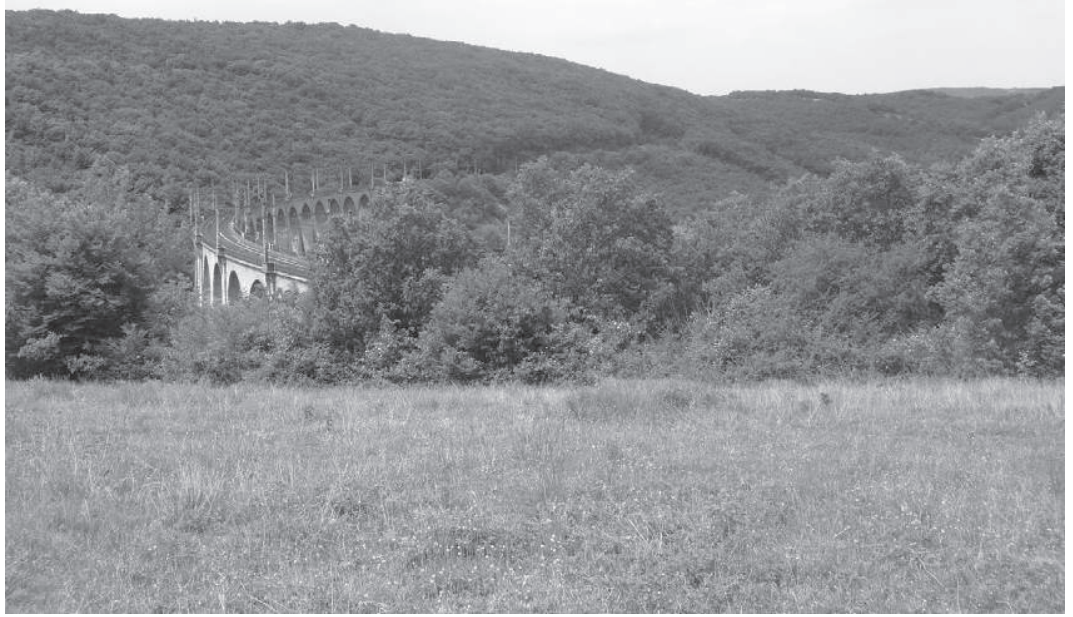

Figure 2. Aux confins du Limousin et du Lot, entre Brive et Souillac, I'artère historique du PO s'inscrit dans un territoire de faible densité et dans un relief difficile. Deux contraintes pour une «ligne TGV ». Cliché auteur.

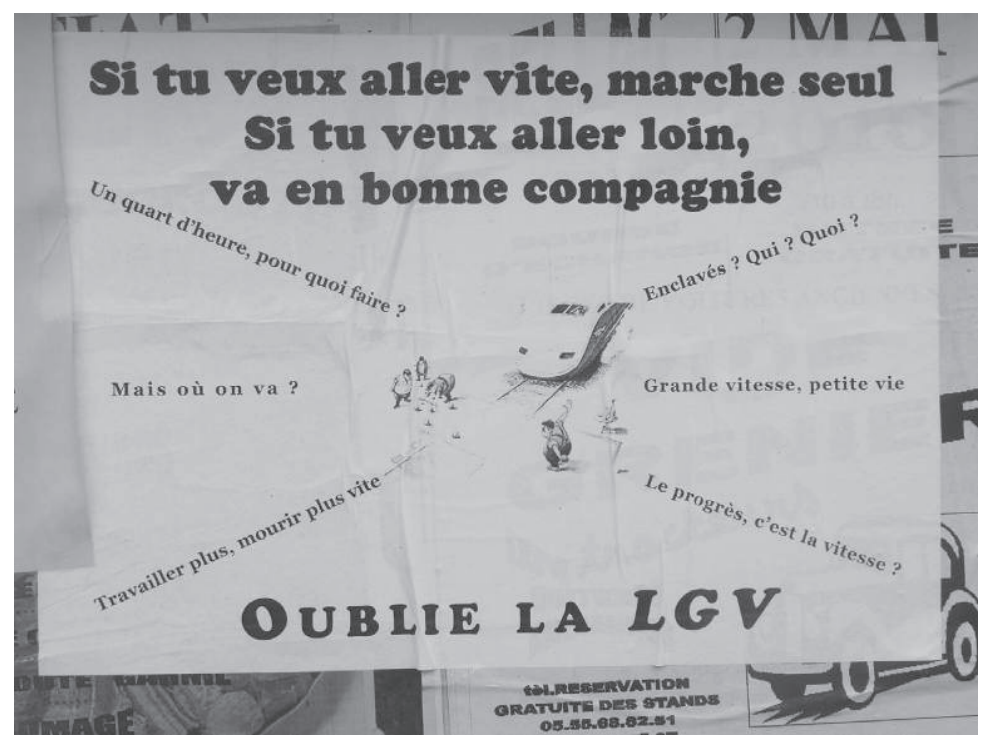

Figure 3. Présentes sur divers supports de l'espace public, ces affiches posent avec poésie et ironie des questions qui invitent à des réflexions fondamentales. Cliché auteur. 


\section{Un troisième rendez-vous encore manqué ? Le projet de LGV Limoges - Poitiers}

Suite à l'échec du TGV POLT apparaît dans la presse ${ }^{16}$ quelques mois plus tard le projet de LGV Poitiers - Limoges dont le coût ne semble guère plus élevé que ne l'était la mise à niveau de l'artère classique pour accueillir le TGV pendulaire. Ce projet, d'abord activement défendu par la femme du Président de la République, Bernadette Chirac, conseillère générale de la Corrèze depuis 1979, a peu à peu remporté l'adhésion d'une large majorité politique allant du PS à l'UMP. Ces partis représentent $80 \%$ de l'électorat limousin et il est intéressant de noter que cette proportion correspond exactement à celle de l'opinion publique se prétendant désormais en faveur de la LGV ${ }^{17}$.

\section{Projet et arguments de la LGV}

Le principe retenu est d'inclure cette infrastructure dans le projet global de LGV "SEA » (Sud Europe Atlantique) qui comprend trois branches ${ }^{18}$, toutes inscrites dans le programme prioritaire de 2000 kilomètres de lignes nouvelles à grande vitesse à lancer avant 2020 (loi du 3 août 2009 relative à la mise en œuvre du Grenelle de l'Environnement). L'objectif affiché est d'irriguer le grand centre-(sud)-ouest et de conforter la dynamique d'ouverture " naturelle » du territoire limousin vers l'Ouest, lui permettant ainsi de profiter pleinement ${ }^{19}$ d'une position géographique privilégiée entre Massif central, façade atlantique et grand Sud-Ouest.

\section{Les opposants : profils et arguments}

Les opposants à la LGV Limoges- Poitiers regroupent en réalité, comme c'est souvent le cas, des catégories de personnes aux motivations et intérêts très variés. On y retrouve les plus directement concernés, à savoir les particuliers et agriculteurs touchés par le futur tracé qui traversera leur propriété. Les représentants des partis politiques traditionnellement anticapitalistes participent ici comme ailleurs à la lutte, de même que les organisations écologistes. Les associations d'usagers du chemin de fer, à travers la FNAUT, expriment eux

16- C'est dans La Vie du rail du 10 mars 2004 qu'il est fait allusion pour la première fois à un courrier de Jean-Pierre Raffarin adressé à Bernadette Chirac, conseillère générale de Corrèze, dans lequel le Premier ministre affirme qu'une ligne à grande vitesse entre Limoges et Poitiers sera mise en service en 2014 et que des études préliminaires seront engagées dès le deuxième trimestre 2004.

17- Enquête menée par RFF en 2009.

18- Tours - Bordeaux - Hendaye, Bordeaux - Toulouse, et Poitiers - Limoges.

19- Limoges - Poitiers en 35 minutes, Limoges - Paris (La Rochelle, Bordeaux) en deux heures. 
aussi leur hostilité au projet. Enfin, plus surprenant ou du moins plus original, le milieu occitaniste trouve ici un terrain d'expression pour évoquer son attachement au "pays ». L'analyse des thèmes des revendications révèle des raisonnements classiques ou plus surprenants. L’argument économique majeur consiste à dire que le projet de LGV serait trois fois plus cher que la «mise à niveau » de la ligne classique. Du point de vue de l'environnement, la dégradation des paysages est évoquée, tout particulièrement celle du site inscrit des monts de Blond. La fracture territoriale est aussi vigoureusement décriée : la LGV marginaliserait un peu plus des espaces déjà fragiles, favorisant une fois encore les pôles urbains. Du point de vue social, se fait jour l'opinion selon laquelle le TGV n'est emprunté que par les "riches». Enfin, une approche que nous qualifierions volontiers de "philosophique " s'exprime à travers des affiches et slogans relevés sur plusieurs communes de Haute-Vienne concernées par le tracé ; à quoi sert la grande vitesse, notre société ira-t-elle mieux en allant plus vite ? C'est en substance la question soulevée... (fig. 3).

À l'heure où les $T G V$ perdent eux aussi de leur rentabilité à tel point que des liaisons ont été supprimées en 2010, à l'heure également où l'État parle d'austérité, est-il certain que le projet de LGV Limoges - Poitiers aboutira malgré le large consensus politique dont il fait désormais l'objet? Si la volonté de l'exécutif régional limousin ne semble pas faiblir, il n'en demeure pas moins évident que les considérations budgétaires l'emporteront probablement et que les arguments des opposants peuvent prendre du poids dans le contexte actuel. Rien n'est donc fait encore une fois pour le Limousin : il est intéressant de constater que les combats d'aujourd'hui sont ceux déjà menés au XIX ${ }^{e}$ siècle.

\section{Lorsque l'histoire se rappelle au présent}

Les divers lobbys qui s'expriment en ce début de $\mathrm{XXI}^{\mathrm{e}}$ siècle démontrent en effet que les idées sont exactement les mêmes que celles qui animaient les investisseurs du chemin de fer voici 150 ans. Deux exemples le prouveront parfaitement.

Le retour des grandes liaisons Atlantique - Europe centrale

L'association ALTRO ("transline ») a pris récemment parti en faveur de la LGV Limoges - Poitiers. Elle considère en effet que la future LGV Limoges Poitiers représente le premier maillon d'un vaste réseau transversal qui ferait de la capitale limousine le point de jonction incontournable vers les grandes villes portuaires de Nantes et Bordeaux quand on vient de l'Europe orientale ${ }^{20}$.

20- Le projet de modernisation de la transversale Lyon - Bordeaux a lui aussi été abandonné en $2003 \ldots$ 


\section{Le retour d'un transpyrénéen central Paris - Madrid}

Le passage du Somport fait aussi parler à nouveau de lui, toujours comme point de liaison stratégique entre Paris et l'Espagne via... Limoges. L'argument est aujourd'hui défendu par l'association «Euro 21 » qui s'est jointe au lobbying " Eurosud-Transport ». Il s'agit de promouvoir à la fois la mise à deux fois deux voies de la route nationale 21 et le développement du fret sur l'axe ferroviaire Paris - Toulouse sur lequel serait embranchée la future liaison transpyrénéenne (que l'on sait incontournable compte tenu de la saturation des voies actuelles). Quelles que soient les options de tracé évoquées, le principe global du projet est bien celui déjà défendu par les acteurs économiques et politiques du XIX ${ }^{\mathrm{e}}$ siècle : il s'agit d'établir un itinéraire Paris - Limousin - Saragosse - Madrid via une traversée centrale des Pyrénées.

\section{Une approche prospective en guise de conclusion}

Ce travail est donc finalement l'occasion de rappeler que l'histoire se répète et que les logiques d'aménagement dépassent les querelles politiques et les visions partisanes qui ont nui au développement "spontané ", ou du moins logique, des infrastructures de transport. Cent à cent cinquante ans plus tard, le Limousin est toujours au coeur des plans de circulation dans l'Europe du Sud-Ouest. Notre dernière carte, qui synthétise les réflexions en cours, témoigne de l'évident rôle potentiel de carrefour de l'espace limousin (fig. 4). Elle témoigne aussi d'une évolution marquée par la métropolisation dont la politique de la grande vitesse représente une remarquable illustration. En Limousin comme ailleurs, les opposants à la LGV dénoncent, au-delà de l'argument environnemental, un outil qui crée comme ailleurs de la fracture territoriale et sociale. On peut en tout cas se demander si les critiques encore récemment développées dans la presse à l'égard du système TGV «à la française » (rames articulées et non sécables, lignes établies trop loin des villes, système trop coûteux expliquant les pertes de marché à l'étranger...) ne marquent pas la fin d'une politique des LGV qui a montré ses limites économiques.

Une chose est certaine : le projet de LGV Poitiers - Limoges reste encore virtuel. Ce qui n'empêche pourtant pas le Limousin de figurer depuis les deux derniers recensements de population parmi les neuf régions les plus attractives de France. Faut-il alors en déduire que la grande vitesse n'est pas une condition pour réussir un développement territorial, qui plus est durable ? Ou faut-il à l'opposé utiliser cet argument démographique pour plébisciter la poursuite du désenclavement limousin qui, avec les infrastructures autoroutières (et aériennes), a sérieusement progressé et autorisé par conséquent un redressement historique? 


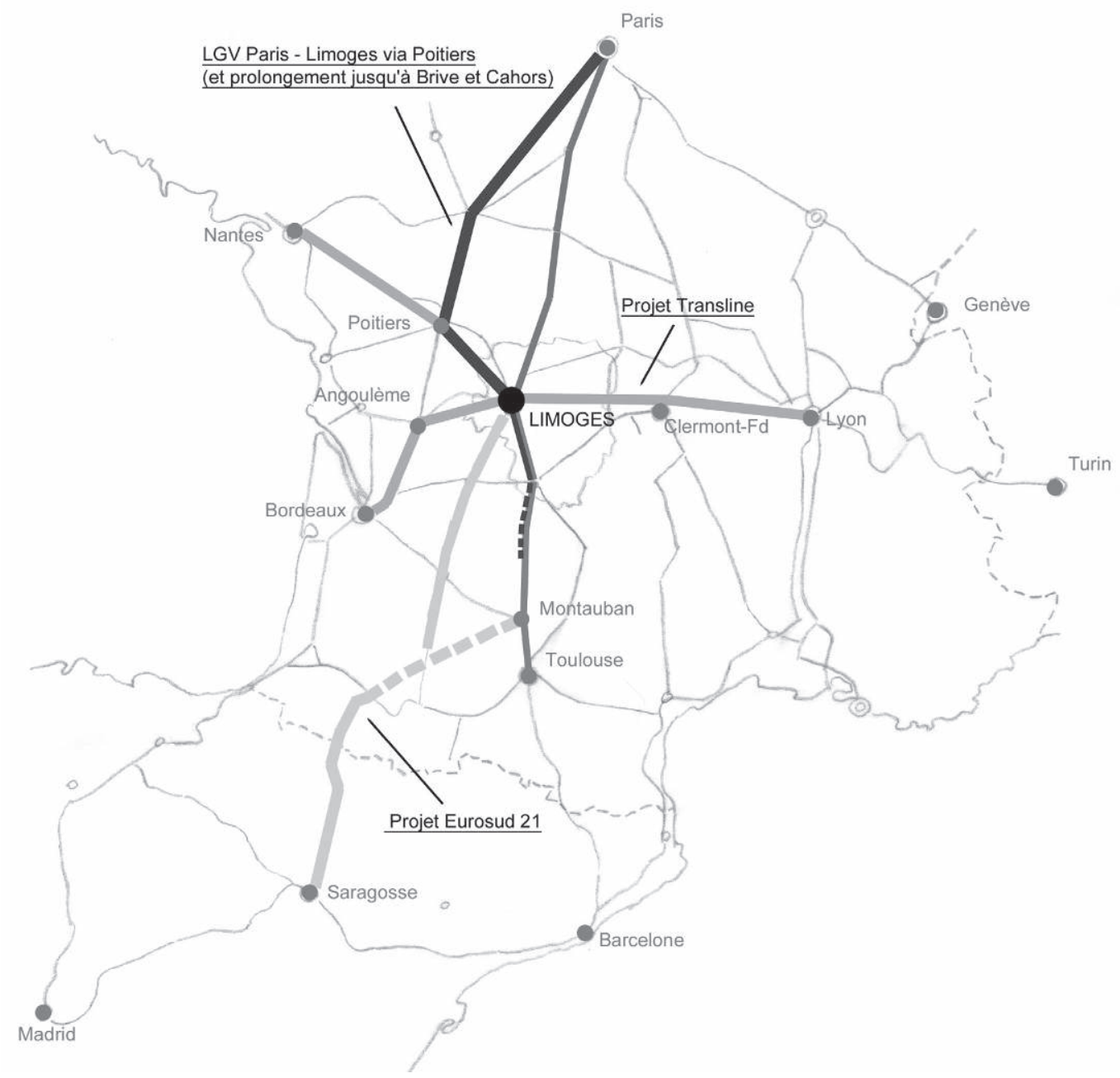

Figure 4. La grande vitesse (fret et voyageurs) en Limousin à I'horizon 2030 ? - Esquisse d'un scénario. - Pascal Desmichel. 


\section{Bibliographie}

[COLL.], 1994 : Atlas du Limousin. Une nouvelle image du Limousin, Limoges, PULIM, 1994, $168 \mathrm{p}$.

[COLL.], 1989 : «Les chemins de fer, l'espace et la société en France ", Actes du colloque des 18-19 mai 1988, Revue d'histoire des chemins de fer hors série, 1, AHICF, 1989, 384 p.

AVENAS et ROUSSET, 1979: Avenas, Jean, Rousset, Jacques, et alii, «Verdoyant et secret Limousin ", La Vie du rail, n 1718 (25 novembre 1979), 60 p.

BANAUDO et LANOUE, 2003 : Banaudo, José et Lanoue, Fabrice, Sur les rails du Limousin, Breil-sur-Roya, Éd. du cabri, 2003, 160 p.

DUPUY, 2000 : Dupuy, Jean-Marc, « 40 années de service pour le « Capitole » ", Rail passion, $\mathrm{n}^{\circ} 44$ (décembre 2000), p. 53-61.

DESMICHEL, 2008 : Desmichel, Pascal, «La gare monumentale de Canfranc à l'épreuve des temps. Grandeur et décadence d'un patrimoine ferroviaire de la montagne aragonaise (Espagne) ", Cybergeo, Aménagement, Urbanisme, article 420, mis en ligne le 14 mai 2008, modifié le 15 mai 2008. URL : http://www.cybergeo.eu/index18342.html.

KAUFFMANN, 2008 : Kauffmann, Vincent, Les Paradoxes de la mobilité. Bouger, senraciner, Lausanne, Presses polytechniques et universitaires romandes, coll. « Le savoir suisse ", 2008, 115 p.

LARTILLEUX, 1956 : Lartilleux, Henri, Géographie des chemins de fer français, t. 1, La SNCF, Paris, Chaix, 1956, 369 p.

LEVY et LUSSAULT, 2003 : Levy, Jacques, et Lussault, Michel (sous la dir.), Dictionnaire de géographie et de l'espace des sociétés, Paris, Belin, 2003, 1034 p.

SNCF, Géographie économique et ferroviaire, t. 3 (cartes), 1966.

VERGEZ-LARROUY, 2001 : Vergez-Larrouy, Jean-Pierre, Les Chemins de fer du PO, Paris / Chanac, La vie du rail / La Régordane, 2001, 320 p.

VEYRINAUD, 1981 : Veyrinaud, Georges, Le Limousin, la nature, les hommes, Limoges, CRDP, 1981, 207 p.

VILAIN, 1960 : Vilain, Lucien-Maurice, Les Chemins de fer de montagne français, t. 1, Vigot Frères, 1960, 178 p.

Sitographie (sites visités le 8 juin 2011)

http://limogespoitiers.com, le site des opposants au projet dit « absurde » de la LGV Limoges-Poitiers.

http://tgv-plb.asso.fr, site de l'association de promotion de la liaison TGV Poitiers - Limoges - Brive. 
http://www.lgvpoitierslimoges.com, le site de Réseau Ferré de France sur le projet de LGV

http://aquitaine.fr/politiques-regionales/transports/transports.html, pages dédiées aux infrastructures de transport dans la région Aquitaine.

http://www.altro.org, site de l'Association logistique transport Ouest (projet transline).

http://www.eurosud-transport.asso.fr, site de l'association Eurosud-transport. 